${ }^{1}$ Comité de Ética, Sociedad Chilena de Nefrología

${ }^{2}$ Facultad de Medicina,

Universidad de los Andes.

${ }^{3}$ Escuela de Enfermería, Pontificia

Universidad Católica de Chile.

${ }^{4}$ Facultad de Medicina,

Universidad Diego Portales.

${ }^{5}$ Facultad de Medicina, Pontificia

Universidad Católica de Chile.

${ }^{6}$ Facultad de Medicina,

Universidad de Chile.

${ }^{7}$ Facultad de Medicina Clínica

Alemana- Universidad de

Desarrollo. Santiago de Chile.

Recibido el 5 de marzo de 2014,

aceptado el 13 de marzo de

2014

Documento aprobado por el directorio de la Sociedad Chilena de Nefrología, con el carácter de documento oficial de esta Sociedad.

Conflictos de intereses: Los autores declararon no tenerlos.

Correspondencia a:

Dr. Antonio Vukusich Covacic.

Clínica Dávila, Recoleta 464

Recoleta, Santiago.

Teléfonos: 27308021

avukusic@davila.cl

\section{Recomendaciones del Comité de Ética de la Sociedad Chilena de Nefrología para el manejo de los problemas ético-clínicos de pacientes adultos con enfermedad renal crónica terminal}

\author{
ANTONIO VUKUSICH ${ }^{1,2}$, MARÍA ISABEL CATONI ${ }^{1,3}$, SOFÍA P. SALAS ${ }^{1,4}$, \\ ANDRÉS VALDIVIESO ${ }^{1,5}$, EMILIO ROESSLER $^{1,6,7}$
}

\section{Recommendations of the Ethics Committee of the Chilean Society of Nephrology for the management of ethical problems in adult end stage renal disease patients}

There are different approaches to treat patients with End Stage Renal Disease (ESRD): hemodialysis, peritoneal dialysis, renal transplantation and conservative medical management. The choice of the best therapy for each patient, needs both clinical and ethical skills. The Ethics Committee of the Chilean Society of Nephrology has elaborated recommendations to help health workers to deal with the ethical and clinical problems related to patients suffering ESRD. Its goal is to guide, at a national level, the effective use of minimal standards in the treatment and care of patients with ESRD, including appropriate care and information for patients, therapy selection, management of difficult cases and potential conflicts.

(Rev Med Chile 2014; 142: 368-374)

Key words: Kidney failure, chronic; Medical ethics; Renal dialysis.
L a enfermedad renal crónica (ERC) tiene una alta prevalencia y el tratamiento de $\checkmark$ su estado terminal (ERCT) suele requerir terapias de sustitución renal (TSR) de alto costo, como la hemodiálisis y la diálisis peritoneal. Las TSR han sido utilizadas con éxito en el mundo desde la década 1960-69. Nuestro país dispuso de cobertura financiera para la hemodiálisis crónica (HDC) sólo a mediados de la década de 1980-89 $\mathrm{y}$, desde entonces, se ha considerado inaceptable restringir estas terapias a un grupo limitado de pacientes, condiciones o patologías y su número ha crecido hasta alcanzar las mejores coberturas de la región.

Las TSR tienen características únicas. Los pacientes deben someterse a ellas de manera permanente y prolongada. El tiempo que consumen obliga a hacer cambios en el estilo de vida, horarios, tipo y sitios de trabajo y, con los años, suelen aparecer otras dolencias y patologías. A pesar de estas limitaciones, los enfermos mantienen una sorprendente adherencia a las TSR, porque han conseguido aumentar la esperanza y calidad de vida de la ERCT y la rehabilitación social e incluso laboral de quienes la padecen. Pero no siempre ocurre así. Con el aumento sostenido de las TSR, debido al aumento de las enfermedades metabólicas y la mayor sobrevida de la patología cardiovascular, se ha puesto en evidencia un grupo de pacientes que no parece beneficiarse. Se trata de pacientes portadores de otras patologías concomitantes que disminuyen la esperanza o 
calidad de vida, y de enfermos muy añosos con limitaciones fisiológicas o secuelas funcionales importantes, que suelen llevar una vida llena de dolencias y carente de sentido personal. Estos pacientes tienen una limitada sobrevida, una pobre calidad de vida y es muy improbable que una TSR cambie su condición; por el contrario, muchas veces es posible predecir con un alto grado de certeza que las consecuencias negativas del tratamiento sustitutivo superarán ampliamente sus beneficios. Para estos pacientes, las TSR pueden llegar a ser un problema más que deben soportar.

Esta situación nos recuerda el ya conocido principio ético de que "no todo lo que se puede hacer, se debe hacer", llamándonos a reflexionar sobre cuándo es apropiado iniciar o continuar una TSR. Una terapia se considera apropiada cuando sus beneficios superan las consecuencias negativas por un margen suficientemente amplio, entendiendo por "beneficios" prolongar la vida y mejorar la calidad de vida de las personas. Para la mayoría de los enfermos las TSR resultan aceptables, adecuadas y salvadoras. Pero pueden transformarse en una terapia desproporcionada, incapaz de cumplir con la finalidad de la medicina: buscar siempre el bien del enfermo, curándolo, cuando es posible, aliviándolo siempre, pero no prolongando artificial e innecesariamente una vida de mala calidad. Cuando un tratamiento sólo consigue prolongar el sufrimiento, agregando más dolor al ya existente, esa terapia cae fuera del sentido mismo de la medicina.

Los pacientes con ERCT no deben ser discriminados para acceder a una TSR por razones de edad, creencias religiosas, ideología, características sociales, económicas, educativas o patología. Sin embargo, una TSR, como cualquier otra, debe estar apropiadamente indicada. Esto ocurre cuando los beneficios superan ampliamente las cargas negativas para el paciente. Existe un amplio consenso en que, desde un punto de vista médico, este es el único criterio válido para seleccionar a los pacientes para una TSR.

Para utilizar de manera apropiada las TSR en la ERCT debemos considerar, además de lo señalado, otros aspectos de gran importancia. En primer lugar, el concepto de ERCT incluye un rango de funciones renales residuales todavía muy diferentes entre sí (clearance de creatinina entre 0 y $15 \mathrm{ml} / \mathrm{min}$ ). Se trata de una variedad de pacientes, con numerosos matices, que el adjetivo "terminal" no recoge. Como consecuencia, se les ofrece a todos ellos el mismo tratamiento: una TSR que, en nuestro medio, suele ser HDC. No se considera la variabilidad funcional de los pacientes, ni se distinguen las características particulares con que cada uno de ellos lleva adelante su enfermedad: el grado de tolerancia y adaptación, la posibilidad de auto cuidado o de adherencia al tratamiento, la co-existencia y pronóstico de otras patologías concomitantes. Las TSR tienen, como cualquier tratamiento, fortalezas y debilidades; por lo tanto, la decisión de cuándo iniciarlas o cuál de ellas utilizar debería ser el resultado de una elección deliberada, en la que han participado tanto el médico como el paciente o sus representantes.

Existen diferentes alternativas para el manejo de la ERCT: HDC, peritoneo diálisis, trasplante renal o tratamiento médico conservador. Antes de elegir alguna, deberíamos mostrar a los pacientes las ventajas, desventajas y pronóstico clínico de cada una de las opciones disponibles. Deberíamos considerar sus creencias y valores, la evaluación personal de los beneficios y cargas que las diferentes alternativas tienen y las metas que el paciente pretende lograr, dependiendo de su capacidad para recibir información, procesarla y tomar decisiones. Todos estos aspectos deberían tener un rol predominante en la elección de la terapia de la ERCT. Tendríamos que contar con procedimientos claros que promuevan la autonomía y participación informada del paciente, o de quienes lo subroguen, en la toma de decisiones. No sólo para la elección de la terapia, sino que a través de todo el proceso de enfermar y morir. Sin embargo, debido a la enorme asimetría de información médico-paciente, la autonomía de este último para consentir o no con un determinado tratamiento resulta con frecuencia, poco practicada y el mismo paciente puede declarar sentirse más cómodo en un ambiente de "paternalismo consentido".

Finalmente, deberíamos considerar el impacto que tienen las decisiones terapéuticas en la ERCT sobre los profesionales que realizan la atención directa. Ellos dedican gran parte de su entrenamiento a la adquisición de destrezas técnico-clínicas necesarias para el manejo de las TSR. Pero también tienen que enfrentar problemas ético-clínicos cuyo manejo requiere de otras habilidades. En la práctica cotidiana suelen aparecer una serie de interrogantes de este tipo: ¿Se debe ofrecer a los pacientes la posibilidad de elegir entre las diferen- 
tes alternativas de tratamiento disponibles para la ERCT? ¿Los pacientes están suficientemente informados para elegir entre ellas? ¿En quiénes se debería iniciar una TSR? ¿Es necesaria una definición de los derechos y deberes específicos de los pacientes con TSR? ¿Debería solicitárseles la firma de una orden de no reanimar para que, en la eventualidad de un paro cardio-respiratorio, el personal sepa a qué atenerse? ¿En qué condiciones es aceptable suspender el tratamiento?

El Comité de Ética de la Sociedad Chilena de Nefrología (SCHN) ha elaborado las siguientes "recomendaciones" como una forma de aportar al manejo de los problemas ético-clínicos de la ERCT. Se consideraron, de manera referencial, diferentes documentos ${ }^{1-17}$ y la publicación de estas recomendaciones cuenta con la aprobación del Directorio de esta Sociedad.

El objetivo de estas recomendaciones es orientar, a nivel nacional, la aplicación efectiva de estándares éticos mínimos en el tratamiento y cuidado de personas con ERCT, incluyendo la atención oportuna, la entrega adecuada de información, la selección de los pacientes y las terapias, el manejo de los casos difíciles y de posibles conflictos. Aunque ellas están dirigidas, primariamente, a los médicos nefrólogos, se espera que constituyan un marco de referencia para la comunidad nefrológica, las autoridades de gobierno relacionadas, los profesionales de la salud que proporcionan el cuidado clínico, la industria que produce y comercializa elementos e insumos para los pacientes con ERCT, y los propios pacientes y sus familias.

\section{Problemas ético-clínicos frecuentes en pacientes adultos con enfermedad renal crónica terminal (ERCT)}

\section{Oportunidad de la atención}

Descripción del problema: La pesquisa y la derivación tardía de los pacientes con enfermedad renal crónica (ERC) avanzada en la atención primaria impiden anticipar la alternativa de tratamiento más adecuada a cada caso. Los pacientes que llegan urémicos y en malas condiciones necesitan un acceso vascular transitorio y hemodiálisis de urgencia.

Recomendación: Desarrolle y mantenga mecanismos de comunicación y coordinación permanentes con la atención primaria para la transfe- rencia oportuna de pacientes con ERC avanzada, evitando así el ingreso de urgencia a hemodiálisis. La derivación oportuna facilita la preparación integral de los pacientes y sus familiares para enfrentar las etapas finales de la ERC y permite la elección de la modalidad de tratamiento más adecuada a cada caso. Participe en instancias de educación a la comunidad. Considere la posibilidad de establecer una consulta pre-dialítica multi profesional para los pacientes que requerirán terapia de sustitución renal (TSR). Ello facilita la adherencia al tratamiento, disminuye la morbimortalidad y puede mejorar la calidad de vida.

\section{Consentimiento informado (I): información al paciente}

Descripción del problema: El equipo tratante prioriza la solución de los aspectos técnico-médicos de la enfermedad y la entrega de información adecuada sobre la ERCT pasa a un segundo plano. Hay una asimetría de información de tal magnitud entre el médico y el paciente-familia que estos últimos no pueden actuar como interlocutores bien informados.

Recomendación: Informe al paciente o su representante de manera oportuna, comprensible y completa el diagnóstico y las diferentes opciones disponibles para el tratamiento de la ERCT: hemodiálisis, peritoneo diálisis, trasplante renal o manejo conservador, con sus respectivos pronósticos. El paciente debe decidir si es necesario involucrar a otras personas de su confianza que podrían ayudarlo a tomar decisiones. Planifique una reunión del paciente y su familia con el equipo de enfermería para abordar algunos aspectos específicos. Valore la competencia del paciente para recibir información, procesarla y tomar decisiones. Entrégueles material informativo comprensible que motive el diálogo entre ellos y con el equipo tratante. Puede suceder que el paciente no esté, transitoriamente, en condiciones psicológicas para recibir una mala noticia con respecto a su salud y que no desee ser informado. Otorgue tiempo al paciente para asimilar su condición antes de entregar una información más completa y tomar las decisiones. Deje registro de estos intentos en la ficha clínica. Los familiares del paciente que ejerzan alguna profesión del área de la salud pueden facilitar la comunicación de conceptos técnicos de difícil comprensión. Cuando sea posible, apóyese también en ellos. 


\section{Consentimiento Informado (II): formalización de la relación}

Descripción del problema: El equipo tratante entrega información al paciente con ERCT pero no queda constancia de ello. La información puede haber sido insuficiente, malinterpretada o no ha llegado, cuando corresponde, a sus representantes.

Recomendación: Deje constancia de la información entregada y de las decisiones tomadas por el paciente o sus representantes, con la firma de un documento de Consentimiento Informado. La firma de este documento es una garantía de que la información se entregó y facilita el establecimiento de una relación que considera al médico $\mathrm{y}$ al paciente como agentes morales capaces de un diálogo eficaz. En los casos que deberán ser tratados con una TSR la consulta pre-dialítica permite, entre otras cosas, anticipar la construcción de un acceso vascular o peritoneal, según corresponda, e iniciar una relación constructiva con el paciente, sus representantes y su familia.

\section{Elección de la terapia}

Descripción del problema: Los pacientes con ERCT presentan diferentes funciones renales residuales $\mathrm{y}$, a pesar de su carácter progresivo, la evolución y las manifestaciones de la enfermedad pueden variar de forma significativa. Todas las alternativas de tratamiento de la ERCT (hemodiálisis, peritoneo diálisis, trasplante o manejo conservador) tienen ventajas y desventajas. Los pacientes competentes o, en su defecto, sus representantes, tienen derecho a participar en la elección del tipo de terapia pero muchas veces no lo hacen.

Recomendación: Promueva una relación médico-paciente-familia que facilite una toma de decisiones compartida. El paciente o su representante deben comprender los límites y el pronóstico de cada tratamiento. La decisión final la deben tomar el paciente o sus representantes, considerando la calidad y esperanza de vida. Esta decisión no será irreversible. Debe quedar abierta la posibilidad de un cambio de opinión y de terapia. Si el paciente presenta otras patologías, también graves y quizás de mal pronóstico, antes de presentarle las alternativas de tratamiento comuníquese con los otros especialistas que lo tratan. Así se podrá establecer en forma más certera la terapia realmente beneficiosa para cada caso y evitar que el paciente reciba distintos mensajes que, aun siendo com- plementarios entre sí, podrían ser interpretados como contradictorios.

\section{Definición de objetivos y aseguramiento de la calidad}

Descripción del problema: En los pacientes con ERCT que inician una TSR no se ha definido previamente, o no se comparte con el paciente o sus representantes, qué, exactamente, se quiere conseguir con el tratamiento y cuando se hace, el personal de salud tiende a fijar objetivos clínicos o de laboratorio y no en términos de calidad de vida del paciente. La terapia no es un fin en sí misma.

Recomendación: Determine los objetivos concretos del tratamiento elegido, teniendo siempre presente que el objetivo primordial es la calidad de vida del paciente, lo que incluye la rehabilitación social y laboral, la carga e intensidad de los síntomas, la tolerancia al tratamiento y el estado psicológico, entre otros. Considere, además, indicadores clínicos y de laboratorio que permitan evaluar la calidad de la TSR. Permita que el paciente y su familia participen en el planteamiento de los objetivos y comparta con ellos los logros y fallas en los resultados esperados. También comparta los objetivos con el personal de salud que asiste al paciente. Lo anterior suma esfuerzos en un mismo sentido y reduce la posibilidad de conflictos al interior del equipo tratante por falta de comunicación.

\section{Selección de pacientes para TSR}

Descripción del problema: No existen restricciones absolutas para iniciar la TSR, pero no se ha evaluado, en cada caso particular, su real utilidad o beneficio ni se ha definido en qué condiciones se planteará su suspensión. El paciente no manifiesta por anticipado sus deseos por medio de un documento ad hoc.

Recomendación: Ningún paciente debiera ser discriminado por razones de edad, nivel educacional, económico o social (incluyendo la utilidad o inutilidad social de una persona), creencias religiosas, ideologías o patologías. Sin embargo, hay condiciones en que el uso de TSR no parece razonable. Se sugiere no iniciar o suspender la TSR en las siguientes condiciones:

- Daño cognitivo severo.

- Estado vegetativo persistente.

- Patología psiquiátrica que impide colaborar con el tratamiento. 
- Cualquier condición en que las cargas o consecuencias negativas del tratamiento superen ampliamente los beneficios.

La decisión de no iniciar o suspender una TSR podría generar problemas éticos a un paciente competente, su familia e incluso al equipo de salud haciendo difícil su materialización, sobre todo cuando hay opiniones divergentes. En pacientes incompetentes, estas decisiones deben tomarse siempre de acuerdo con sus representantes, teniendo presente el sentido que tiene el tratamiento en cada caso. Con el fin de facilitar el proceso para la familia y el equipo de salud, toda vez que se pueda, estimule tempranamente a los pacientes a compartir lo que desean que se haga cuando ya no estén en condiciones de tomar decisiones. La existencia de un documento que manifieste, por anticipado, la voluntad del paciente facilita la toma de decisiones y la posibilidad de que reciba el cuidado que desea. Su implementación asegura el respeto a la autonomía del paciente o su derecho a la autodeterminación del tratamiento que desea recibir. Este documento podrá ser modificado por el paciente en cualquier momento. Se sugiere iniciar su aplicación, de manera personalizada, y utilizando para este fin la ficha clínica del paciente.

\section{Inicio de la TSR en casos dudosos}

Descripción del problema: Hasta un tercio de los pacientes que llegan a desarrollar una ERCT reúne condiciones que hacen dudar a la familia o al equipo tratante sobre si una TSR traerá más beneficios que consecuencias negativas. Este es el caso de los pacientes de edad avanzada, con múltiples enfermedades y limitaciones funcionales, en los que no es seguro que una TSR mejore la sobrevida y la calidad de vida.

Recomendación: Inicie, a modo de prueba y de común acuerdo con el paciente o sus representantes, la TSR más probablemente beneficiosa. Precise con ellos qué objetivos se deberían conseguir con la TSR para decidir su mantención y cuánto tiempo durará el ensayo. Recuerde que el objetivo más importante es mejorar la calidad de vida. Pueden necesitarse semanas o meses de observación antes de que el paciente competente o sus representantes tomen una decisión definitiva. Aunque la decisión corresponde finalmente siempre al paciente o sus representantes, es recomendable que ella sea con- versada y compartida por todos: paciente, familia y equipo tratante.

\section{Retiro de la TSR}

Descripción del problema: Los profesionales clínicos asisten a pacientes que ya no parecen beneficiarse con la TSR. La familia manifiesta sus dudas y temores en la eventualidad de la suspensión de la terapia. Suspender la TSR en la ERCT es difícil para la familia y el equipo tratante y la decisión tiende a postergarse.

Recomendación: La decisión de suspender definitivamente la TSR debería basarse en la calidad de vida conseguida con el tratamiento o en que la prolongación de la vida -a pesar de una mala calidad de vida- tenga algún sentido para el paciente. Se trata de una decisión personal del paciente, si es competente, o de sus representantes si no lo es. El retiro de la TSR puede ser progresivo o absoluto, completo o parcial. Se sugiere proporcionar el tratamiento considerando tanto las condiciones y necesidades específicas de cada paciente como su tolerancia a los cambios. El uso de ultrafiltración aislada ocasional puede mantener al paciente libre de los temidos síntomas de una sobrecarga hidrosalina. Antes de suspender o disminuir la terapia informe claramente sobre los cambios en la dieta y en la terapia medicamentosa que serán necesarios. Deben explicarse los síntomas o problemas que podrían presentarse y cuál sería la mejor manera de proceder para cada uno de ellos. El equipo tratante debe estar disponible para reevaluar la situación cada vez que sea requerido y aceptar una vuelta atrás por el cambio de opinión del paciente o sus representantes. Es recomendable contar con protocolos de cuidados paliativos que permitan dar continuidad a la atención de los pacientes que suspenden (o no se incorporan) a la terapia dialítica. Promueva la vinculación eficaz del paciente con un sistema adecuado de cuidados paliativos.

\section{Manejo conservador de la ERCT}

Descripción del problema: La amplia disponibilidad de TSR ha dejado al tratamiento médico conservador de la ERCT en un segundo plano. Sin embargo, algunos pacientes con ERCT optan por no iniciar una TSR.

Recomendación: No iniciar una TSR es una decisión personal basada en la calidad de vida que se desea tener. Asegúrese que el paciente competente haga esta elección de manera libre e informada. 
Descarte una depresión, estrés o síntomas intolerables que al ser tratados podrían modificar la decisión del enfermo. Por otra parte, entre los pacientes con ERCT encontramos todavía un amplio rango funcional renal $y$, en pacientes añosos dependiendo del clearance residual, el tratamiento médico conservador ha demostrado su superioridad tanto en cantidad como calidad de vida. Protocolice un manejo conservador, ordenado y preciso de cada uno de los problemas de la ERCT que serán abordados sin TSR. Utilice guías simples que sirvan de orientación y apoyo permanente a las personas que cuidarán al enfermo. En cada caso informe cuidadosamente al paciente o sus representantes acerca de la necesidad de agregar los cuidados paliativos que corresponda, cuando llegue el momento.

\section{Abordaje de conflictos}

Descripción del problema: Las TSR de la ERCT determinan una forma de vivir con dependencia absoluta de la tecnología. El paciente podría no aceptar el inicio de una TSR o decidir su retiro aun cuando ella sea aparentemente beneficiosa. Esto puede generar conflictos entre el paciente o sus representantes con el equipo tratante; pero también entre los distintos profesionales que asisten al enfermo. Por otra parte, el paciente podría no adaptarse, ser poco responsable con su cuidado o tener conductas inadecuadas. Los pacientes poco colaboradores o no adherentes al tratamiento deterioran sus relaciones con el equipo tratante.

Recomendación: No improvise. Establezca un procedimiento formal, sistemático y preciso para resolver los conflictos que se podrían presentar con los pacientes, sus familias o el equipo a cargo. Disponga de un canal expedito para ofrecer una segunda opinión al paciente o sus representantes en caso de dudas o pérdidas de confianza. Busque la asesoría de un Comité de Ética para los casos sin acuerdo. Nunca haga cambios en la TSR que se está realizando hasta que se resuelvan los conflictos. En el caso de un paciente incompetente, si el médico o el equipo tratante no están de acuerdo con la decisión de sus representantes, pueden tomar medidas, previa consulta al Comité de Ética que corresponda, en pro de la defensa del paciente ya sea para que continúe con vida o para permitirle fallecer. Para el equipo de salud siempre debe estar primero el mejor interés del paciente. De otra manera se estaría reconociendo, tácitamente, que la decisión de los representantes del paciente está por sobre la integridad moral de los profesionales de la salud que lo asisten.

\section{Referencias}

1. Ardiles L, Poblete H, Ortiz M, Elgueta S, Cusumano A, Vukusich A, Mezzano S. The Health System in Chile: The Nephrologist Perspective. J Nephrol 2011; 24 (02): 149-54.

2. Lelie A, Dekkers W, van Hamersvelt H, Huysmans F, ten Have H. Discontinuing dialysis: patient's wishes and professional judgement. Nephrol Dial Transplant 1999: 14: 318-21.

3. Holley J, Carmody S, Moss A, Sullivan A, Cohen L, Block S, et al. The Need for End-of-Life Care Training in Nephrology: National Survey Results of Nephrology Fellows. Am J Kidney Dis 2003; 42 (4): 813-20.

4. Andreucci V, Kerr D, Kopple J. Rights of chronic renal failure patients undergoing chronic dialysis therapy. Personal Opinion Nephrol Dial Transplant 2004; 19: 30-8.

5. Davison S, Jhangri G, Holley J, Moss A. Nephrologists' Reported Preparedness for End-of-Life DecisionMaking. Clin J Am Soc Nephrol 2006 (1): 1256-62.

6. Morey-Molina A, Rodríguez-Jornet A, Monfá-Bosch J, Torquet-Escuder P, González-Álvarez M, SánchezCasajús A, et al. Ética y nefrología. Consensos de la Sociedad Española de Diálisis y Trasplante. Dial Traspl 2006; 27 (3): 102-7.

7. Conservative treatment: Choosing not to start dialysis. The Kidney Foundation of Canada. 2007. (www.kidney. ca).

8. Visser A, Dijkstra G, Kuiper D, de Jong P, Franssen C, Gansevoort R, et al. Accepting or declining dialysis: considerations taken into account by elderly patients with end-stage renal disease. J Nephrol 2009; 22 (6): 794-9.

9. Cooper B, Branley P, Bulfone L, Collins J, CraigJ, Fraenkel M, et al. Randomized, Controlled Trial of Early versus Late Initiation of Dialysis (IDEAL Study). N Engl J Med 2010; 363: 609-19.

10. Shared Decision-Making in the Appropriate Initiation of and Withdrawal from Dialysis. Renal Physicians Association Clinical Practice Guideline. Rockville, Maryland 2010.

11. Moss A. Ethical Principles and Processes Guiding Dialysis Decision-Making. Clin J Am Soc Nephrol 2011; 6: 2313-7.

12. Sánchez-Tomero J, Rodríguez-Jornet A, Balda S, Cigarrán S, Herrero JC, Maduell F, et al. Exploring the opinion of CKD patients on dialysis regarding end-of- 
Life \& Advance Care Planning. Nefrología 2011; 31 (4): 449-56.

13. Germain M, Davison S, Moss A. When Enough Is Enough: The Nephrologist's Responsibility in Ordering Dialysis Treatments. Am J Kidney Dis 2011; 58 (1): 13543.

14. Davison S. The Ethics of End-of-Life Care for Patients with ESRD. Clin J Am Soc Nephrol 2012; 7: 2049-57.

15. Wulff HR, Pedersen SA, Rosenberg R. La dimensión ética de las decisiones médicas. En: Introducción a la filosofía de la medicina. Triacastela, Madrid, 2002.

16. León F. Ley de derechos y deberes de las personas en la atención de salud. Una mirada bioética. Rev Med Chile 2012; 140: 1490-4.

17. Muthalagappan S, Johansson L, Kong WM. Dialysis or conservative care for frail older patients: ethics of shared decision-making. Nephrol Dial Transplant 2013; 28: 2717-22. 